\title{
Transição dos cuidados de pacientes com doenças crônicas da pediatria para a medicina de adultos: práticas de um hospital terciário no Brasil
}

\section{Transition to adult health care for adolescents with chronic conditions: practices of a tertiary care hospital in Brazil}

Patricia Souza Valle Cardoso Pastura ${ }^{1}$ e Caroline Graça de Paiva ${ }^{1}$

'Fundação Oswaldo Cruz

\section{Palavras-chave:}

Transição para assistência do adulto; Cuidado transicional; Adolescente; Doença crônica; Serviços de saúde.

\section{Resumo}

Introdução: devido aos avanços nos cuidados médicos, 90\% das crianças com doenças crônicas estão, na atualidade, sobrevivendo até a vida adulta. Por este motivo, é necessário um processo de transição dos cuidados da pediatria/hebiatria para a medicina do adulto que envolva uma equipe multidisciplinar e seja planejado, individualizado, gradual e baseado nas competências do adolescente, principalmente nos seus próprios cuidados e no conhecimento da sua doença. Objetivo: avaliar as condições da transição implementadas em serviços de especialidades pediátricas de um hospital público materno-infantil terciário no Rio de Janeiro. Métodos: estudo descritivo qualitativo baseado na aplicação de um questionário específico para avaliação da organização dos serviços de especialidades pediátricas quanto a este processo de transição de cuidados. Resultados: em apenas três de cinco unidades avaliadas existe um profissional ou uma equipe de profissionais de saúde dedicados ao processo de transição, porém não exclusivamente. Em nenhuma das cinco unidades avaliadas há consulta conjunta entre o pediatra e o médico de adultos antes da transferência, apesar de existirem referências externas para três serviços. Nenhum serviço utiliza ferramentas validadas para auxiliar a avaliação das habilidades do adolescente ao longo do processo ou conta com programas educativos específicos para a transição. Conclusões: não há um programa estabelecido de transição de cuidados para a medicina de adultos para pacientes com doenças crônicas em nossa unidade.

\section{Keywords:}

Transition to adult care; Transitional care; Adolescent; Chronic disease; Health services.

\begin{abstract}
Introduction: due to advances in medical care, $90 \%$ of children with chronic diseases are currently surviving into adulthood. For this reason, a process of transition, involving a multidisciplinary team, from pediatric/hebiatric care to adult medicine is necessary. This process should be planned, individualized, gradual and based on the teenager's skills, especially on his/her own care and on the knowledge of his/her disease. Objective: to evaluate the transition conditions implemented in some pediatric specialities units from a public tertiary child hospital in Rio de Janeiro. Methods: qualitative descriptive study consisting on the application of a specific questionnaire to evaluate the organization of pediatric specialities services regarding the transition from pediatric medicine to adult care. Results: only three of five evaluated units have a professional or a team of health professionals dedicated to the transition process, but not exclusively. None of the five units evaluated have a joint consultation with the pediatrician and the adult physician prior to the transition, although there are external references for three of them. No service utilizes validated tools to assess adolescent skills throughout the process or implements educational programs for the transition. Conclusions: there is not an established transition program of care to adult medicine for patients with chronic diseases in our hospital.
\end{abstract}




\section{INTRODUÇÃO}

A prevalência da doença crônica na população infantil é de aproximadamente $20 \%$, considerando condições físicas, deficiências no desenvolvimento, dificuldades de aprendizagem e doença mental. ${ }^{1-3}$ Dentre as inúmeras doenças crônicas da infância, podemos destacar fibrose cística, cardiopatias congênitas, paralisia cerebral e câncer.

O termo 'doença crônica' tem várias definições na literatura, caracterizando-se primariamente pela progressão e necessidade de tratamentos prolongados. Além do curso longo, pode também ser definida por ser incurável, deixar sequelas ou impor limitações às funções do indivíduo, requerendo adaptação contínua. ${ }^{4}$

Em uma revisão, Moreira e colaboradores ${ }^{4}$ destacaram também o comprometimento das dimensões de sociabilidade específicas da infância (dias de ausência na escola, limitações das atividades cotidianas e atraso nos marcos do desenvolvimento) associado a vulnerabilidade e necessidade de apoio para as funções humanas de interação, comunicação, expressão ou necessidade de suporte tecnológico à vida.

Assim, a doença crônica impõe modificações na vida de crianças/adolescentes e suas famílias, exigindo readaptações e estratégias para seu enfrentamento e suporte às necessidades. Nesse sentido, torna-se essencial o acompanhamento da família e da criança durante todo o processo do tratamento, através de um vínculo de confiança e segurança na equipe médica. Tão importante quanto o tratamento da doença em si é a atenção dada aos aspectos sociais da doença. ${ }^{5}$ As relações que as crianças estabelecem com seus cuidadores e a sensibilidade destes em identificar aspectos de atenção, escuta e acolhida podem estabelecer circuitos de troca positivos durante o tratamento e a hospitalização.

Os avanços nos cuidados de saúde nas últimas décadas conduziram ao aumento da sobrevida de pacientes pediátricos com doenças crônicas e condições complexas de saúde, chegando estes até a vida adulta. ${ }^{3,4,6,7}$ Esta mudança epidemiológica, que também tem sido observada no Brasil, ${ }^{6}$ se por um lado significa melhoria de indicadores sociais e de saúde, por outro, individualmente, implica na necessidade de um processo de gestão da passagem dos cuidados pediátricos para a medicina do adulto, garantindo uma abordagem eficaz e sensata.

O processo de preparação e transferência da criança com doença crônica que é acompanhada há anos em um serviço de pediatria para a saúde de adultos deve começar no início da adolescência e envolve planejamento individualizado e contínuo, incluindo aspectos da gestão de ambos os setores hospitalares ou da rede de saúde, bem como competências do próprio adolescente. ${ }^{3}$ A mudança do ambiente seguro da pediatria, no qual a equipe de saúde mantém uma relação de longo prazo com o paciente e com a família, para a medicina de adultos pode configurar-se um problema para os adolescentes portadores de doenças crônicas., ${ }^{5,-9}$

Então, um processo eficaz de transição de cuidados é um elemento necessário no cuidado da criança com doença crônica e, atualmente, o conceito de transição planejada é considerado parâmetro de qualidade nos serviços que prestam cuidados a estes pacientes. ${ }^{10} \mathrm{E}$ importante distinguir transferência - entendida como um ato único de transferir o doente de uma instituição ou equipe de cuidados a outra - e transição, definida como um processo dinâmico, complexo e planejado, que inclui a avaliação da aquisição de habilidades e competências, assim como a implementação de programas educacionais e recursos de cuidados de saúde. O objetivo da transição é "maximizar o funcionamento e o potencial ao longo da vida através do fornecimento de serviços de saúde de alta qualidade e adequados ao desenvolvimento que continuam ininterruptos à medida que o indivíduo se move da adolescência para a idade adulta".

O processo de transição deve começar cedo, no início da adolescência, para que haja tempo adequado de preparar a família, os médicos e o adolescente antes deste atingir a idade adulta. Embora as opiniões sobre a idade para o início desse processo divirjam, recomenda-se iniciá-los ao redor dos 12 anos de idade, mas pode haver benefícios em iniciar as discussões sobre a transição mais cedo ou mais tarde.,

Na verdade, o momento da transferência para o atendimento de adultos deve basear-se menos na idade e mais na aquisição de habilidades individuais do paciente, incluindo a prontidão e a capacidade de negociar com o sistema de saúde de adultos. ${ }^{11,12}$ Deve-se levar em consideração a época em que a doença foi diagnosticada, bem como as transformações que acarreta na vida do indivíduo, englobando processos de tomada de decisão e construção de uma rede que abranja família, hospital, escola e sistema de garantia de direitos. ${ }^{13,14}$ Também deve-se considerar se os adolescentes exibem 'comportamentos adultos', como gravidez, abuso de substâncias ilegais, desafio às autoridades. Eles tendem a ser desligados dos serviços pediátricos precocemente, às vezes, sem os cuidados inerentes à transição.

Dentre as consequências da falha de transição nas diferentes condições crônicas estão o aumento da rejeição de órgãos, o aumento da hemoglobina glicada 
em jovens com diabetes mellitus tipo 1 e o aumento da atividade da doença em patologias reumatológicas ou imunomediadas. $^{8}$

Ao redor dos anos 2010, em alguns países, formaram-se grupos de estudo dos problemas relacionados à transição, e houve a elaboração de recomendações, programas e ferramentas para auxiliar profissionais de saúde, adolescentes e famílias a enfrentarem o processo. ${ }^{15-18}$ Nos EUA, surgiu o Got Transition/Center for Health Care Transition Improvement (Centro para a Melhoria de Transição de Cuidados de Saúde), que introduziu o conceito Six Core Elements of Health Care Transition (Seis Elementos Essenciais de Transição de Cuidados de Saúde). ${ }^{19}$

Estes componentes incluem: 1) estabelecimento de uma política que habilite os adolescentes no desenvolvimento da independência e autocuidado, iniciando-se entre os 12 e os 14 anos; 2) acompanhamento do progresso da transição a partir dos 12 anos; 3 ) avaliações de prontidão para a transição através de ferramentas padronizadas; 4) planejamento para a transição aos cuidados de adultos por meio da identificação das prioridades dos adolescentes, especialmente para tomada de decisões, a partir dos 16 anos; 5) transferência e integração nos cuidados do adulto com um histórico médico e também um plano de cuidados de atendimento de emergência; 6) conclusão da transferência na perspectiva de cuidados contínuos, com mecanismos de feedback da experiência do jovem.

Dentre as várias ferramentas práticas para uso em cuidados primários e especializados que auxiliam o processo da transição de cuidados da pediatria para a medicina de adultos, destacamos o Transition Readiness Assessment Questionnaire (TRAQ), elaborado e validado entre 2007 e 2008, na Flórida, Estados Unidos. ${ }^{20,21}$ Este questionário mede as habilidades necessárias para o sucesso da transição, assim como o progresso dos adolescentes em outras áreas, como educação, trabalho e vida diária. É composto de dois domínios, sendo que o primeiro avalia competências de autogestão (interpretação de prescrições, compreensão de efeitos colaterais do tratamento, manipulação de equipamentos médicos, agendamento de consultas e questões de gestão financeira da saúde) e o segundo, competências para autoadvocacia (comunicação com profissionais da saúde e gestão das atividades da vida diária, incluindo uso de recursos da escola e da comunidade). O TRAQ acaba de ser traduzido e validado no Brasil. ${ }^{22}$

O objetivo do presente artigo é avaliar as condições da transição de cuidados para a medicina de adultos de pacientes com doenças crônicas acompanhados em cinco diferentes serviços de especialidades pediátricas de um hospital público terciário materno-infantil na Cidade do Rio de Janeiro, Brasil.

\section{MÉTODO}

Trata-se de um estudo descritivo qualitativo, com a utilização de um questionário autoaplicável a gestores de serviços de especialidades pediátricas que acompanham pacientes crônicos até a adolescência em um hospital público terciário, materno-infantil na Cidade do Rio de Janeiro.

O questionário foi escolhido por ter sido desenvolvido em português e aplicado na Cidade do Porto, Portugal, em um trabalho semelhante, de 2011. ${ }^{23}$ Não havia intenção de que as respostas ao questionário determinassem, através de alguma métrica, quais serviços realizam um 'programa de transição de cuidados' adequadamente. Na verdade, algumas perguntas abertas também foram respondidas pelos gestores, em uma entrevista. Estes foram eleitos por serem os que mais conhecem os processos e particularidades dos atendimentos de cada serviço de especialidade pediátrica do hospital.

Foi solicitado que excluíssem considerações acerca do processo de transição de pacientes que não chegarão a ter autonomia por deficiência cognitiva grave, dentre aqueles acompanhados pela neurologia e genética especialmente. Os gestores também assinaram o Termo de Consentimento Livre e Esclarecido, após aprovação do projeto no Comitê de Ética e Pesquisa local com Certificado de Apresentação para Apreciação Ética número 62721716.1.0000.5269.

\section{RESULTADOS}

Foram obtidos cinco questionários preenchidos pelos responsáveis dos serviços pediátricos de pneumologia, infectologia, gastroenterologia/nutrologia, neurologia e genética médica, cujos resultados encontram-se na tabela 1. Estes são os serviços que atendem grande quantidade de adolescentes com doenças crônicas, diferentemente das especialidades de alergia e dermatologia, não incluídas. As principais doenças crônicas acompanhadas por esses serviços são: síndrome da imunodeficiência adquirida (Sida), fibrose cística, doença pulmonar crônica, doenças cromossômicas, doenças desmielinizantes, transtorno do espectro autista, síndromes epilépticas, doenças de má absorção intestinal, desnutrição e obesidade.

Analisando as respostas, verifica-se que em nenhuma especialidade há um 'programa de transição' 
Tabela 1 - Resultados do questionário sobre a transição de cuidados da pediatria para medicina de adultos em serviços de um hospital terciário (2017)

\begin{tabular}{|c|c|c|c|c|c|}
\hline PERGUNTAS & Neurologia & Infectologia & Pneumologia & Genética & Nutrição/Gastro \\
\hline $\begin{array}{l}\text { 1- Existe um profissional ou uma equipe de profissionais de } \\
\text { saúde dedicados ao processo de transição? }\end{array}$ & NÃO & SIM & SIM & SIM & NÃO \\
\hline $\begin{array}{l}\text { 2- O plano de transição é implementado no início da adoles- } \\
\text { cência (11-14 anos) e posteriormente revisto com o jovem, } \\
\text { entre os 14-18 anos? }\end{array}$ & NÃO & - & NÃO & NÃO & NÃO \\
\hline $\begin{array}{l}\text { 3- Há encorajamento para que os adolescentes intervenham } \\
\text { no programa de transição? }\end{array}$ & SIM & SIM & - & SIM & NÃO \\
\hline $\begin{array}{l}\text { 4- Antes da transferência, há realização de algumas consultas } \\
\text { conjuntas (pediatra e médico do adulto)? }\end{array}$ & NÃO & NÃO & $\begin{array}{c}\text { SERÁ } \\
\text { IMPLEMENTADO }\end{array}$ & NÃO & NÃO \\
\hline $\begin{array}{l}\text { 5- O adolescente é estimulado a ter conhecimentos sobre a } \\
\text { sua patologia e determinação das aptidões que são necessá- } \\
\text { rias adquirir, estimulando a sua autonomia? }\end{array}$ & SIM & SIM & SIM & SIM & SIM \\
\hline 6- O jovem tem oportunidade de ir às consultas sem os pais? & SIM & SIM & SIM & SIM & SIM \\
\hline $\begin{array}{l}\text { 7- Faculta-se o acesso a livros ou revistas sobre as dificuldades } \\
\text { encontradas pelos jovens com patologia crônica? }\end{array}$ & SIM & SIM & SIM & NÃO & SIM \\
\hline $\begin{array}{l}\text { 8- Há preocupação em garantir que se mantenha a multidisci- } \\
\text { plinaridade assistencial no processo de transição, obtendo } \\
\text { um trabalho de cooperação entre todos os elementos } \\
\text { que o constituem (pediatra, médico do adulto, assistente } \\
\text { social...)? }\end{array}$ & SIM & SIM & - & SIM & SIM \\
\hline $\begin{array}{l}\text { 9- Há a preocupação de envolver o médico de família nesse } \\
\text { processo de transição? }\end{array}$ & SIM & NÃO & NÃO & SIM & SIM \\
\hline $\begin{array}{l}\text { 10- Há facilidade de acesso à informação médica entre os médi- } \\
\text { cos que tratam o jovem? }\end{array}$ & SIM & SIM & SIM & NÃO & SIM \\
\hline 11- Durante o processo de transição são discutidos: & & & & & \\
\hline $\begin{array}{l}\text { 11.1- os problemas de saúde de forma que o adolescente e a } \\
\text { família compreendam a doença? }\end{array}$ & SIM & SIM & SIM & SIM & SIM \\
\hline 11.2- mudança de papéis do jovem e família? & SIM & SIM & $\mathrm{SIM}$ & SIM & SIM \\
\hline 11.3- promoção e educação para a saúde? & SIM & SIM & $\operatorname{SIM}$ & SIM & NÃO \\
\hline $\begin{array}{l}\text { 11.4- problemáticas de ordem social, psicológica (incluindo } \\
\text { autoestima), comunicacional, vocacional e da área sexual? }\end{array}$ & SIM & SIM & $\begin{array}{l}\text { SERÁ } \\
\text { IMPLEMENTADO }\end{array}$ & SIM & SIM \\
\hline $\begin{array}{l}\text { 11.5- estabelecimento de planos de futuro (formação acadê- } \\
\text { mica e profissional)? }\end{array}$ & SIM & SIM & $\begin{array}{c}\text { SERÁ } \\
\text { IMPLEMENTADO }\end{array}$ & SIM & SIM \\
\hline 11.6- diferenças dos serviços de adulto e pediátrico? & SIM & SIM & NÃO & NÃO & SIM \\
\hline 11.7- visita ao serviço de adultos e seu agendamento? & NÃO & NÃO & $\begin{array}{c}\text { SERÁ } \\
\text { IMPLEMENTADO }\end{array}$ & NÃO & NÃO \\
\hline
\end{tabular}

implementado. Não há consulta conjunta entre pediatras e clínicos ou especialistas de adultos, antes do momento da transferência, nem visita prévia dos adolescentes ao novo serviço/hospital. Também não são utilizadas ferramentas para avaliação de habilidades do adolescente ao longo do processo. Apesar de existir multidisciplinaridade assistencial - médicos, fisioterapeutas, psicólogos, assistentes sociais e nutricionistas - em todos os serviços, o que acaba por auxiliar no processo, apenas em três existe um profissional ou uma equipe de profissionais dedicados ao processo de transição, porém não exclusivamente.

Em todas as unidades são discutidos os problemas de saúde de forma que o adolescente compreenda a doença, as mudanças de papéis na família durante a adolescência e a transição para a medicina do adulto. $\mathrm{O}$ adolescente é estimulado a adquirir autonomia, incluindo a possibilidade de ir à consulta sem os pais.

Em quatro das cinco especialidades avaliadas, discutem-se problemas de ordem social, psicológica, 
comunicacional, vocacional e da área sexual, bem como o estabelecimento de planos para o futuro do jovem, incluindo assuntos sobre formação acadêmica e profissional.

O serviço de neurologia direciona seus pacientes para hospitais de adultos através do Sistema Nacional de Regulação (Sisreg). Este processo tem sido bastante falho, pela demora ou dificuldade de se estabelecer acordos com outras unidades de saúde de adultos. Algumas vezes, é o contato pessoal que garante a inserção de pacientes portadores de doenças desmielinizantes em ambulatório especializado de um hospital federal regional. É comum encontrarmos pacientes com mais de 21 anos ainda assistidos no serviço.

Na infectologia pediátrica, os pacientes portadores de HIV/Aids começam a ser direcionados para outras unidades ao completarem 18 anos. Como a maioria dos casos é de transmissão vertical, os adolescentes preferem dar continuidade ao tratamento no mesmo local em que as mães o fazem. Quando isso não ocorre, eles já são automaticamente transferidos para o Instituto Nacional de Infectologia, que conta com uma agenda específica para esses jovens, com a vantagem da manutenção do contato entre si, na sala de espera de suas consultas. Na verdade, muitos deles trocam ideias em redes sociais, alguns que já foram transicionados ajudando os que estão no processo. Os gestores desse serviço enfatizaram a questão do estímulo à autonomia, em conversas sobre tomada de decisão, autocuidado, administração de medicamentos e sexualidade.

No serviço de genética quase todos os pacientes, ao completarem 21 anos, são direcionados para um dos hospitais universitários da cidade. A transferência é feita através de laudos médicos e acordos já estabelecidos entre os médicos geneticistas de ambos os hospitais; sem, contudo, ser discutido com os pacientes as diferenças entre os serviços pediátrico e de adulto. Os adolescentes portadores de osteogênese imperfeita em tratamento com pamidronato são encaminhados a um hospital de referência em endocrinologia.

Na nutrologia, os pacientes acompanhados por desnutrição ou obesidade são encaminhados à clínica de saúde da família próxima à sua residência, através de laudos médicos, por meios próprios ou via Sisreg. Já os pacientes portadores de doença celíaca, fibrose cística, doença intestinal inflamatória ou outras síndromes disabsortivas são direcionados para hospitais terciários com serviço de gastroenterologia, também via Sisreg. Não há idade-limite de acompanhamento e a média na transferência é de 19 anos. Alguns pacientes com doenças em estágios avançados permanecem no serviço além dessa idade, requerendo empenho pessoal dos médicos-assistentes para sua inserção em unidades de adultos.

Os pacientes com fibrose cística são o principal grupo de adolescentes da pneumologia, geralmente atendidos desde o início da infância e mantendo, portanto, forte vínculo com toda a equipe de saúde. A adolescência representa uma fase crítica do tratamento, não só no que concerne aos medos, angústias e incertezas próprios da idade e quanto ao momento de transferência, mas, sobretudo, pela conscientização crescente em relação à sua menor expectativa de vida. Pelo risco de abandono do tratamento e agudizações, em todas as consultas o adolescente é estimulado a ter conhecimento sobre sua doença, a importância da fisioterapia respiratória (exercícios de mobilização de secreções) e o uso das medicações contínuas, nos devidos horários. O assunto 'transição' ou 'transferência', entretanto, não é devidamente abordado nas consultas, assim como não há um plano específico que os prepare para serem absorvidos por outro hospital. Eles/elas permanecem na especialidade até 18 a 21 anos, quando são transferidos para um dos dois serviços especializados na doença entre adultos, em hospital federal ou universitário.

\section{DISCUSSÃO}

O estudo de Martins ${ }^{23}$ de 2011, na Cidade do Porto, que gerou o questionário sobre adequação do processo de transição entre serviços de especialidades pediátricas, identificou que apenas em duas unidades existia um profissional ou uma equipe de profissionais de saúde dedicados ao processo. Em ambas, a transição de cuidados foi considerada efetiva - um processo contínuo, supervisionado e revisado periodicamente. Diferentemente dos resultados obtidos por Martins, em nosso hospital, avaliando outras especialidades, exceto a pneumologia em comum, atualmente não existe um programa de transição implementado.

No país, a discussão sobre transição de cuidados da pediatria para a medicina de adultos ainda é incipiente, com raros trabalhos de especialidades pediátricas, que ainda não caracterizam a utilização de protocolos

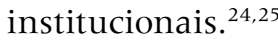

Este não é um problema apenas dos países de língua portuguesa, porém. A discussão nos Estados Unidos, por exemplo, já avança há 20 anos aproximadamente, com o primeiro consenso sobre transição (Academia Americana de Pediatria/Academia Americana de Médicos de Família/Colégio Americano de Médicos/Sociedade Americana de Medicina Interna) datado de 2002. ${ }^{7}$ 
Inicialmente, em 2005, uma pesquisa no mesmo país revelou que apenas 50\% dos responsáveis por adolescentes com doenças crônicas discutiram o processo de transição para a medicina de adultos com o hebiatra. ${ }^{3}$ Em 2008, entre centros de fibrose cística, constatou-se que menos da metade executavam de forma consistente avaliações de transição, e menos de $20 \%$ tinham um programa para promover o desenvolvimento de competências de autogestão e conhecimento para a transição entre os adolescentes. ${ }^{26}$ Ainda atualmente, em nosso centro de referência para fibrose cística não há avaliações consistentes para o processo de transição, apesar de discussão constante com adolescentes e familiares sobre a doença, estimulando a autonomia e autogestão.

Já entre os anos de 2011 e 2012, um trabalho descreveu o processo de transição dos portadores de doenças crônicas em cinco hospitais de Colúmbia, após a implementação do programa baseado nos Seis Elementos Essenciais de Transição de Cuidados de Saúde. ${ }^{27}$ Os adolescentes entre 14 e 24 anos receberam aulas sequenciais, individuais ou em grupo, de preparo para a transição e foram monitorados em sua evolução através de questionários de prontidão. Conforme avançavam na capacidade de autonomia e aquisição de novas habilidades, ficavam cada vez mais próximos à transferência para os cuidados de adultos.

No Brasil, em 2016, Machado e colaboradores descreveram a implementação da Clínica de Transição do Adolescente (CTA) entre portadores de HIV/Aids, a partir dos 16 anos de idade. O programa implementado no local tem três componentes: o uso de parâmetros específicos para avaliar a prontidão para a transição, consultas com os médicos de adultos ainda na CTA e, após os 18 anos, consultas na clínica de adultos concomitantemente com duas a três remarcações na CTA. Este estudo, qualitativo, também descreveu as experiências, sentimentos e entendimentos dos próprios adolescentes durante o processo.

Em nosso estudo, podemos pensar que a transição dos adolescentes portadores de HIV/Aids é facilitada, não pela organização do serviço pediátrico, mas por haver um centro de referência regional para tratamento de adultos infectados que acolhe os adolescentes após 18 anos, mantendo o vínculo social entre eles.

Outro trabalho nacional foi um inquérito eletrônico com reumatologistas pediátricos inscritos na Sociedade Brasileira de Reumatologia. Dentre os que responderam ( $68 \%$ do total), só $13 \%$ utilizam um programa bem-estabelecido de transição de cuidados, e $83 \%$ não utilizam ferramentas para avaliar a prontidão para a transição.
Quarenta e oito porcento dos respondedores mantêm maiores de 18 anos em seus serviços.

Na Alemanha, um estudo publicado em 2016 relatou a experiência de entrevistas de avaliação das competências e das necessidades de 29 adolescentes com condições crônicas, que culminou na elaboração de um programa educacional. ${ }^{28}$ Foram identificadas lacunas nos cuidados e os temas de reflexão sobre a transição selecionados incluíram: novo papel como paciente, orientação no sistema de saúde, questões vocacionais, desapego dos pais, apoio social, contracepção, abuso de substâncias, planejamento familiar, gestão do estresse e desenvolvimento de metas pessoais. Concluiu-se que programas em grupo promovem a autogestão e o preparo dos adolescentes para a transição.

De fato, os programas de educação para a transição dos cuidados, como o relatado na Alemanha ou aquele implementado nos Estados Unidos (o Got Transition), têm se mostrado eficazes; seja através de palestras, seja por meio de cursos, individuais ou coletivos, seja ainda através de grupos de jovens. ${ }^{19,28}$ Os assuntos devem ser discutidos de forma a facilitar o entendimento da doença, orientar o autocuidado e estimular o adolescente a adquirir autonomia e autoestima, estabelecendo expectativas realistas quanto ao futuro. ${ }^{7,18}$ Não observamos nenhuma iniciativa dessa natureza no nosso estudo.

A implementação de uma política de transição não é fácil. Destacam-se entre os principais obstáculos: falta de coordenação de cuidados entre a pediatria e a clínica de adultos; falta de uma equipe exclusiva para o processo; deficiências na infraestrutura, particularmente nos locais para adultos; problemas quanto à alocação dos recursos de saúde; resistência dos adolescentes em desvincularem-se do pediatra-assistente; e idade tardia no início da transição. ${ }^{27}$ Outra limitação de alguns programas de transição está na ausência de um questionário que avalie a aptidão dos adolescentes para desvincularem-se da pediatria e serem tratados na medicina do adulto. ${ }^{9,16-17}$ Consideramos a falta deste instrumento para o acompanhamento individual dos adolescentes com doenças crônicas um importante marcador da ausência de programas formais de transição de cuidados para a medicina de adultos em nosso hospital.

Uma revisão sistemática francesa de 2017 analisou a metodologia empregada em 39 programas de transição de cuidados e o uso de questionários validados foi relatado em apenas $28 \%$ deles. ${ }^{29}$ Outro achado desta revisão foi a falta de indicação da idade na transferência em 
15 estudos. Esses dados reforçam a ausência de consenso mundial sobre questões inerentes ao processo transicional, como validação de questionários e idade-limite.

Outra revisão, de 2016, da Cochrane concluiu pela dificuldade em estabelecer a efetividade dos programas de transição e sugeriu que mais trabalhos sejam realizados voltados a esta temática. ${ }^{30}$

\section{CONCLUSÕES}

Através deste trabalho, pudemos verificar que ainda não existe, dentre as várias especialidades que tratam adolescentes com doenças crônicas na nossa unidade, um programa estabelecido de transição de cuidados da pediatria para a medicina de adultos.

\section{REFERÊNCIAS}

1 Perrin JM, Bloom SR, Gortmaker SL. The increase of childhood chronic conditions in the United States. JAMA. 2007;297(24):2755-9.

2 Kitsantas P, Kornides ML, Cantiello J, Wu H. Chronic physical health conditions among children of different racial/ethnic backgrounds. Public Health. 2013;127(6):546-53.

3 Scal P, Ireland M. Addressing transition to adult health care for adolescents with special health care needs. Pediatrics. 2005;115(6):1607-12.

4 Moreira MCN, Gomes R, Sá Calheiros MR. Doenças crônicas em crianças e adolescentes: uma revisão bibliográfica. Ciênc Saúde Coletiva. 2014;19(7):2083-94.

5 Bauman LJ, Drotar D, Leventhal JM, Perrin EC, Pless IB. A review of psychosocial interventions for children with chronic health conditions. Pediatrics. 1997;100(2):244-51

6 Moreira MEL, Goldani MZ. A criança é o pai do homem: novos desafios para a área de saúde da criança. Ciênc Saúde Coletiva. 2010;15(2):321-7.

7 American Academy of Pediatrics, American Academy of Family Physicians, American College of Physicians, American Society of Internal Medicine. A consensus statement on health care transitions for young adults needs. Pediatrics. 2002;110(Supl 3):1304-6.

8 Gortmaker SL, Walker DK, Weitzman M, Sobol AM. Chronic conditions, socioeconomic risks, and behavioral problems in children and adolescents. Pediatrics. 1990;85(3):267-76.

9 Lotstein DS, McPherson M, Strickland B, Newacheck PW. Transition planning for youth with special health care needs: results from the National Survey of Children with Special Health Care Needs. Pediatrics. 2005;115(6):1562-8.

10 Nunes P, Sassetti L. Transferência ou transição? A passagem da pediatria para a medicina de adultos. Saúde Infantil. 2010;32:116-9.

11 Reiss JG, Gibson RW, Walker LR. Health care transition: youth, family, and provider perspectives. Pediatrics. 2005;115(1):112-20.

12 Rosen DS, Blum RW, Britto M, Sawyer SM, Siegel DM. Transition to adult health care for adolescents and young adults with chronic conditions: position paper of the Society for Adolescent Medicine. J Adolesc Health. 2003;33(4):309-11.

13 Kennedy A, Sloman F, Douglass JA, Sawyer SM. Young people with chronic illness: the approach to transition. Intern Med
Apesar da escassez de dados nacionais, podemos pressupor que esta é também a realidade de muitos outros hospitais no Brasil.

Por outro lado, talvez este trabalho tenha marcado o início de uma discussão interna, no sentido de que cada serviço procure implementar um programa específico, conforme já está estabelecido na literatura, que conte com atividades educacionais, protocolos e aplicação de instrumentos/ferramentas que avaliem os estágios de domínio para as habilidades necessárias, individualmente, aos adolescentes no processo de transição.

Sugerimos que estas medidas possam também ser revistas em outras unidades da nossa cidade e do país, de maneira geral.
J. 2007;37:555-60.

14 Beal SJ, Riddle IK, Kichler JC, Duncan A, Houchen A, Casnellie L et al. The associations of chronic condition type and individual characteristics with transition readiness. Acad Pediatr. 2016;16(7):660-7.

15 American Academy of Pediatrics; American Academy of Family Physicians; American College of Physicians; Transitions Clinical Report Authoring Group, Cooley WC, Sagerman PJ. Supporting the health care transition from adolescence to adulthood in the medical home. Pediatrics. 2011;128(1):182-200.

16 Crowley R, Wolfe I, Lock K, McKee M. Improving the transition between paediatric and adult healthcare: a systematic review. Arch Dis Child. 2011;96(6):548-53.

17 Cramm JM, Strating MMH, Nieboer AP. The role of team climate in improving the quality of chronic care delivery: a longitudinal study among professionals working with chronically ill adolescents in transitional care programmes. BMJ Open. 2014;4:e005369.

18 Canadian Paediatric Society. Transition to adult care for youth with special health care needs. Paediatr Child Health. 2007;12(9):785-8

19 Got Transition. Six Core Elements of Health Care Transition 2.0: integrating young adults into adult health care for use by internal medicine, family medicine, and med-peds providers [access on 10 Feb 2017]. Available from: https://www.gottransition.org/resourceGet.cfm?id=212

20 Zhang LF, Ho JS, Kennedy SE. A systematic review of the psychometric properties of transition readiness assessment tools in adolescents with chronic disease. BMC Pediatr. $2014 ; 9 ; 14: 4$

21 Sawicki GS, Lukens-Bull K, Yin X, Demars N, Huang IC, Livingood $\mathrm{W}$ et al. Measuring the transition readiness of youth with special healthcare needs: validation of the TRAQ - Transition Readiness Assessment Questionnaire. J Pediatr Psychol. 2011;36(2):160-71.

22 Anelli CG, Len CA, Terreri MT, Russo GCS, Reiff AO. Validation of a Brazilian Portuguese version of the transition readiness assessment questionnaire (TRAQ) in a population of transitional youth with chronic rheumatologic disorders. J Pediatr (Rio J). 2018; pii:S0021-7557(17)30800-8.

23 Martins C. Transição dos cuidados médicos pediátricos para a medicina de adultos. Porto. Dissertação [Mestrado em Medicina] - Instituto de Ciências Biomédicas Abel Salazar/ Centro Hospitalar do Porto; 2002 [acesso em 7 nov 2016]. 
Disponível em: https://sigarra.up.pt/fmdup/pt/pub_geral. show_file?pi_gdoc_id=597325

24 Machado DM, Galano E, de Menezes Succi RC, Vieira CM, Turato ER. Adolescents growing with HIV/AIDS: experiences of the transition from pediatrics to adult care. Braz J Infect Dis. 2016;20(3):229-34.

25 Anelli CG, Amorim ALM, Osaku FM, Terreri MT, Len CA, Reiff A. Challenges in transitioning adolescents and young adults with rheumatologic diseases to adult care in a developing country - the Brazilian experience. Pediatr Rheumatol Online J. 2017;15(1):47.

26 McLaughlin SE, Diener-West M, Indurkhya A, Rubin H, Heckmann R, Boyle MP. Improving transition from pediatric to adult cystic fibrosis care: lessons from a national survey of current practices. Pediatrics. 2008;121(5):el160-6.

27 McManus M, White P, Barbour A, Downing B, Hawkins
K, Quion N et al. Pediatric to adult transition: a quality improvement model for primary care. J of Adolesc Health. 2015;56(1):73-8.

28 Bomba F, Herrmann-Garitz C, Schmidt J, Schmidt S, Thyen $\mathrm{U}$. An assessment of the experiences and needs of adolescents with chronic conditions in transitional care: a qualitative study to develop a patient education programme. Health Soc Care Community. 2017;25(2):652-66.

29 Le Roux E, Mellerio H, Guilmin-Crépon S, Gottot S, Jacquin P, Boulkedid R et al. Methodology used in comparative studies assessing programmes of transition from paediatrics to adult care programmes: a systematic review. BMJ Open. 2017;7:e012338.

30 Campbel F, Biggs K, Aldiss SK, O'Neill PM, Clowes M, McDonagh J et al. Transition of care for adolescents from pediatric services to adult health services. Cochrane Database Syst Rev. 2016;4:CD009794. 